\title{
Archaebactéries et évolution
}

Les archaebactéries constituent-elles les fossiles vivants des premiers âges de la vie terrestre?

\section{Carlo Cocito}

Professeur de microbiologie générale et de génétique moléculaire, Faculté de médecine, Université de Louvain, Bruxelles.

\section{Remerciements}

Les données récentes décrites dans cet article sont issues du "FEMS Symposium on Evolution of Prokaryotes " (Federation European Microbiological Societies, Munich, FRG, I6-I8 september 1984). L'auteur est reconnaissant au Comité organisateur pour l'invitation au Symposium.

\section{ADRESSE}

C. Cocito : Unité de microbiologic générale et de génćtique moléculairc, faculté de médecine, ICP, UCL 7449, avenue Hippocrate 75, B-I 200 Bru- es études de la taxonomie (qui classent les êtres vivants selon leur degré de parenté) et celles de l'évolution (qui analysent l'origine des différents organismes) ont été bouleversées, au cours de notre siècle, par les approches expérimentales apportées par la biologie moléculaire. De sciences morphologiques qu'elles étaient à l'origine, elles sont devenues des disciplines biochimiques et ont largement contribué à donner une base moléculaire à l'évolution des espèces.

\section{Taxonomie des protistes}

Le classement des êtres vivants selon les critères de la mobilité et de la capacité photosynthétique avait abouti à l'établissement des royaumes des plantes et des animaux, en laissant les microbes apatrides. Le royaume des protistes fut introduit ensuite par Haeckel dans le but de caser les organismes microscopiques dépourvus de fonctions d'ensemble et de morphogenèse, deux attributs des êtres supérieurs. Des études ultérieures révélèrent la présence, au sein des protistes, de deux types d'organisa- tion cellulaire : l'une, pluricompartimentale (ou eucaryote), l'autre, monocompartimentale (ou procaryote). Les protistes supérieurs ou eucaryotes possèdent un noyau et des organelles cytoplasmiques (mitochondries, chloroplastes, lysosomes, appareil de Golgi) pourvus de membranes, alors que ces compartiments sont absents chez les procaryotes. Les protistes supérieurs furent ensuite divisés en protophytes (organismes proches des plantes), protozoaires (organismes proches des animaux) et histérophytes (organismes de type intermédiaire), ct les protistes inférieurs en eubactéries (Schizomyces) (pour la plupart non photosynthétiques) et en cyanobactéries (Schizophyces) (capables de photosynthèse avec libération d'oxygène). Sur ces bases, un premier arbre de l'évolution fut construit : il était basé sur des ressemblances morphologiques des êtres vivants, ainsi que sur le développement de formes de vie plus élaborées (eucaryotes) à partir de formes moins évoluées (procaryotes) (figure I $A$ ).

Ce premier arbre généalogique fut modifié plus récemment, à la suite de la mise en évidence des appareils génétiques des organelles cyto- 
plasmiques, une découverte qui avait insufflé une nouvelle vie à l'ancienne théorie endosymbiotique. En fait, chloroplastes et mitochondries possèdent un appareil génétique propre de type bactérien, c'està-dire un génome constitué d'ADN seul (dans les chromosomes nucléaires, on retrouve de l'ADN lié à des ARN et à des protéines), des ribosomes $70 \mathrm{~S}$ (les ribosomes du reticulum endoplasmique sont de type $8 \circ \mathrm{S}$ ), des polymérases, des ARNt et également des aminoacylARNt synthétases différentes des polymères cytoplasmiques correspondants $[\mathbf{I}, 2]$.

Sur la base de ces études, un nouveau schéma fut proposé.

Un ancêtre commun à tous les organismes vivants aurait surgi en premier lieu sur notre planète : il s'agit d'un protocaryote anaérobie. Les capacités photosynthétiques et respiratoires se seraient développées ultérieurement en donnant lieu aux bactéries actuelles (eubactéries et cyanobactéries). L'origine des eucaryotes (urcaryote) aurait eu lieu dans une étape ultérieure, grâce à l'établissement de symbioses stables entre bactéries anaérobies (protoeucaryotes), bactéries aérobies (mitochondries) et cyanobactéries (chloroplastes) [3-6]. Ces théories aboutirent à la construction d'un nouvel arbre évolutif qui fut valable jusqu'en I 977 ( figure I B).

\section{Découverte} des archaebactéries

L'étude actuelle de l'évolution est basée sur l'emploi de marqueurs phylogénétiques qui se doivent d'être complexes, indispensables et universels en même temps. Les seuls types de molécules ayant les qualités requises sont les facteurs génétiques (les gènes et leurs expressions phénotypiques), lesquels se sont révélés, en fait, les meilleurs " outils" pour ce genre d'étude [7]. L'information que ces hauts polymères peuvent nous fournir étant proportionnelle aux détails structuraux qui en ressortent, c'est le développement de techniques de pointe pour l'analyse des fragments macro-moléculaires d'abord, et de leurs séquences ensuite, qui a permis de dévoiler la structure fine des protéines en un premier temps, puis celle des ARN, et finalement celle des ADN. Ces travaux ont abouti à la découverte d'un nouveau royaume, celui des archaebactéries, et ont permis la construction de nouveaux arbres évolutifs.

Les ribosomes $70 \mathrm{~S}$ des procaryotes sont formés de 2 sous-unités, l'une petite $(30 \mathrm{~S})$, l'autre grande $(50 \mathrm{~S})$ : la première étant engagée dans l'initiation des chaînes peptidiques, et la seconde dans l'élongation. Chaque unité est constituée d'ARNr et de protéines-r. Plus exactement, la petite sous-unité des ribosomes d' $E$. coli renferme un $\mathrm{ARNr}$ i $6 \mathrm{~S}$ et 2 I protéines, et la grande sous-unité deux $\mathrm{ARNr} 5 \mathrm{~S}$ et $23 \mathrm{~S}$, ainsi que 32 protéines [8-10]. Chaque molé-

cule d'ARNr ou de protéine-r est unique : chaque structure a donc été sélectionnée au cours de l'évolution (environ $3 \times 10^{9}$ années, sur les $4,5 \times 10^{9}$ années d'existence de notre planète) comme étant la plus efficiente des nombreuses possibles. L'étude de la structure fine de ces molécules pourrait donc nous fournir des renseignements précieux quant à la parenté des différents organismes, à leur évolution, et aux propriétés d'ancêtres communs.

Ce concept a guidé les travaux de Woese et ses collaborateurs, auxquels on doit la découverte des archaebactéries. L'approche choisie a été celle de l'analyse comparative des ARNr I6S de microorganismes issus d'habitats singu-

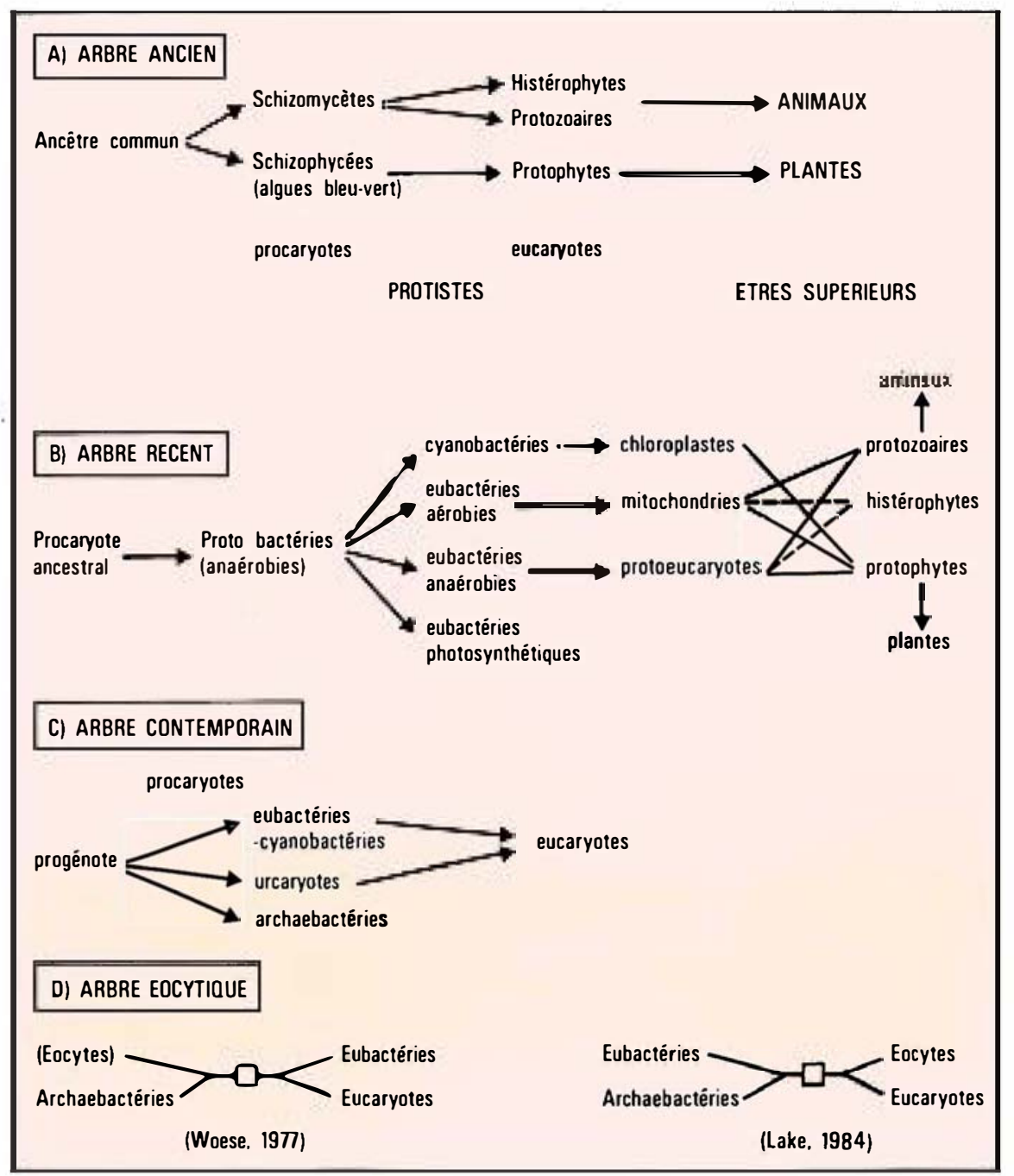

Figure 1. Arbres de l'évolution. 


\section{RÉFÉRENCES}

I. Gibor A, Granick S. Plasmids and mitochondria: inheritable systems. Science 1964; 145: 8907

2. Preer JR Jr. Extrachromosomal inheritance: hereditary symbionts, mitochondria, chloroplasts. Annu Rev Genet 1971; 5: 361-406.

3. Cohen SS Are/Were mitochondria and chloroplasts micro-organisms? American Scientist 1970; 58: $281-9$.

4. Margulis L. Symbiosis and evolution. Sci Am I971; 225 : 49-57.

5. Cohen SS. Mitochondria and chloroplasts revisited. American Scientist 1973; 6r: 437-45

6. Schwartz RM, Dayhoff MO. Origins of prokaryotes, eukaryotes, mitochondria, and chloroplasts. Science 1978; 199: 395-403.

7. Wilson AC, Carlson SS, White TJ. Biochemical evolution. Ann Rev Biochem 1977; 46: 573639

8. Wittmann HG, Components of bacterial ribosomes. Annu Rev Biochem 1982; 51: 155-83.

9. Lake JA, Evolving ribosome structure: domains in archaebacteria, eubacteria, and eucaryotes. Cell 1983; 33: 318-9.

10. Wittmann HG, Architecture of prokaryotic ribosomes. Annu Rev Biochem 1983; 52: 35-65.

II. Woese CR, Sogin M, Stahl D, Lewis BJ, Bonen L. A comparison of the $16 S$ ribosomal RNAs from mesophilic and thermophilic Bacilli: Some modifications in the Sanger method for RNA sequencing. 7 Mol Evol 1976; 7. 197-213.

12. Fox GE, Magrum LJ, Balch WE, Wolfe RS, Woese CR. Classification of methanogenic bacteria by $16 \mathrm{~S}$ ribosomal RNA characterization. Proc Natl Acad Sci USA 1977; 74: 4537-4I.

13. Fox GE, Pechman KJ, Woese CR. Comparative cataloging of $16 \mathrm{~S}$ ribosomal ribonucleic acid: molecular approach to procaryotic systematics. Int 7 Syst Bacteriol 1977; 27: 44-57.

14. Magrum LJ, Luehrsen KR, Woese CR. Are extreme halophiles actually "bacteria"? I Mol Evol 1978; I 1: I-8.

15. Zeikus JG. The biology of methanogenic bacteria. Bacteriol Rev 1977; 4I: 514-4I. liers, sur la base du raisonnement suivant. Puisque les conditions actuelles de vie sur la terre (oxygène, photosynthèse, cycles du carbone, de l'azote et du soufre) sont absolument différentes de celles d'il y a trois milliards d'années, c'est dans des " niches " à caractère primitif que l'on pourrait espérer retrouver les survivants des organismes ancestraux.

L'ARNr i6S est une molécule monocaténaire d'environ I 500 ribonucléotides, qui est enroulée sur elle-même de façon à former une structure secondaire complexe, grâce à l'appariement de bases complémentaires (figure 2). Cela comporte l'établissement de domaines de la molécule, grâce à une alternance de boucles et de tiges : la parenté entre organismes est établie sur la base de la conservation de ces structures. Par exemple, le clivage de cette molécule par la ribonu- cléase $T_{\text {I }}$ produit une série de fragments pouvant être séparés par chromatographie et par électrophorèse : d'où la possibilité de comparaison de fragments issus d'organismes différents. Ce type d'analyse a été appliqué à l'étude de différents procaryotes, mais aussi d'eucaryotes, qui possèdent un $\mathrm{ARNr}$ I8 $\mathrm{S}$ dans la petite sous-unité des ribosomes cytoplasmiques, et des $\mathrm{ARNr}$ I6 $\mathrm{S}$ dans les ribosomes chloroplastiques et mitochondriaux. L'analyse des résultats ainsi obtenus a révélé l'existence d'un groupe d'organismes, situé à part tout à la fois des eucaryotes et des procaryotes conventionnels. Woese et ses collaborateurs ont appelé ces organismes aberrants "archaebactéries", pour indiquer qu'il s'agissait d'une sorte d'ancêtre des bactéries actuellement existant sur la terre [I I-I4]. Aujourd'hui, à la suite de nombreux travaux, le concept d'archaebactérie

\section{* GLOSSAIRE *}

Paroi bactérienne : (uracil), $\mathrm{OH}$ en $\mathrm{C}_{6}$ et $\mathrm{CH}_{3}$ en $\mathrm{C}_{5}$ L'enveloppe est constituée de plusieurs (thymine). Dans les nucléotides corhaut-polymères interconnectés, qui respondants (cytidine, uridine et thypossèdent des structures différentes midine), le $C_{1}$ d'une molécule de dans les différents organismes et sont, ribose forme un lien glycosidique avec dès lors, d'une grande valeur taxono- le $\mathcal{N}_{1}$ pyrimidinique; dans la mique. La couche de base, le peptido- pseudouridine le lien se fait avec le glycane, est constituée d'un poly- $C_{5}$ de l'uracile.

saccharide linéaire fait d'unités alter-

nées $d$ 'N-acétyl-glucosamine et de Lipides membranaires :

son ester lactyl, l'acide muramique. Dans une partie des lipides de tous A ce dernier est relié un tétrapeptide les organismes, les groupements alcool dont un des acides aminés possède du glycérol sont engagés dans un lien deux groupements $\mathrm{NH}_{2}$ (lysine ou ester avec les groupements carboxyles son dérivé bicarboxylique, acide des acides gras. Dans les archaebactédiaminopimélique), ce qui permet ries, un lien type éther est établi entre l'édification d'un réseau tridimension- groupements alcool du glycérol d'une nel responsable de la résistance du part et des alcools méthylés (phytapeptidoglycane. La couche suivante nols) d'autre part.

est représentée par l'acide teichoïque

ou l'arabino-galactanemycolate dans Séquences de fixation :

les Gram-positif, et par un Au niveau de l'ADN : le promoteur lipopolysaccharide-polypeptide pour l'attachement de l'ARN poly(endotoxine) dans les Gram-négatif. mérase (synthèse d'ARN messager); Bases pyrimidiniques : polypuriniques Shine-Dalgarno $A$ l'hexahétérocycle pyrimidinique $\left(5^{\prime} \ldots A G G A G G \ldots 3^{\prime}\right)$ qui précèdent porteur d'un $\mathrm{OH}$ en $\mathrm{C}_{2}$ sont de 4 à 7 bases le codon d'initiation attachées les fonctions suivantes: $A U G$, et qui fixent le ribosome (tra$\mathrm{NH}_{2}$ en $\mathrm{C}_{6}$ (cytosine), $\mathrm{OH}$ en $\mathrm{C}_{6}$ duction du message en protéines). 


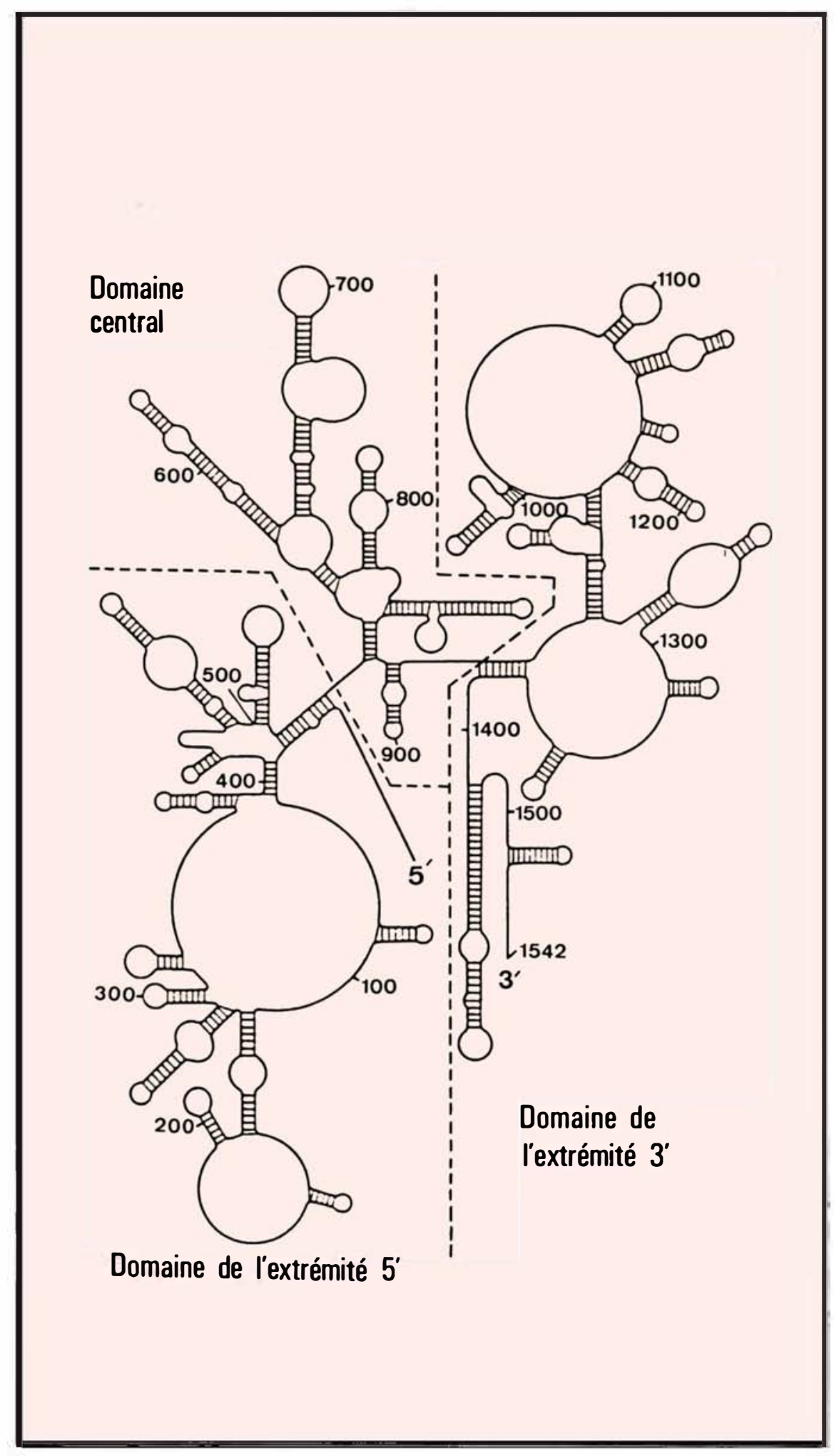

Figure 2. Structure primaire de l'ARNr de la petite sous-unité ribosomique bactérienne. L'établissement de la séquence en bases de l'ARNr $16 \mathrm{~S}$ a permis l'élaboration d'une structure complexe formée par des "boucles" monocaténaires et des "tiges " bicaténaires (appariement $A-U$ et G-C par des ponts hydrogène). Le schéma de la molécule avec ses trois domaines sert de référence pour les études phylogénétiques.

$m / s n^{0} 8$, vol. 2, octobre 86 peut être considéré comme étant définitivement acquis.

\section{Propriétés biologiques et classification}

Une caractéristique commune à toutes les archaebactéries est leur distribution écologique insolite : il s'agit d'habitats interdits à la plupart des bactéries et cyanobactéries conventionnelles. Les propriétés de ces " niches" sont celles qui paraissent avoir existé lorsque la vie s'est établie sur la terre : la classification des archacbactéries reflète ces propriétés écologiques. Les archaebactéries peuvent être divisées en trois grands groupes $\left[\mathrm{I}_{5}\right]$ :

(a) les méthanogènes sont des anaérobies strictes produisant la dégradation de substrats organiques et la formation d'hydrocarbures. En présence d' $\mathrm{H}_{2}$, ils sont capables de réduire le $\mathrm{CO}_{2}$ et les substrats à I carbone (I C) (notamment le formate) pour produire du méthane selon une voie métabolique unique. On les retrouve sur le fond des océans et des lacs, mais également dans les intestins et le rumen des animaux.

(b) les halophyles extrêmes sont des aérobies se développant dans des bassins à haute concentration saline (Grand Lac Salé, Mer Morte, bassins d'évaporation d'eau de mer). Ces organismes sont incapables de vivre hors d'un habitat halophyle (solutions salines proches de la saturation), car ils se servent du gradient osmotique pour le transfert de molécules à travers la membrane cellulaire.

(c) les thermoacidophiles sont des aérobies qui vivent dans des sources thermales très acides. Ils poussent à des températures proches du point d'ébullition de l'eau et à des $\mathrm{pH}$ proches de $\mathrm{I}$, et ils se servent d'un gradient de $\mathrm{pH}$ pour le flux transmembranaire de précurseurs. Certains organismes de ce groupe ont un métabolisme dépendant du soufre.

Quatre types morphologiques d'archaebactéries ont été décrits : (a) formes allongées; (b) formes sphériques isolées; (c) formes en paquets de sphères (sarcina); (d) formes spirillaires. Par ailleurs, on a trouvé des archaebactéries 
Gram-positives et Gram-négatives, chimiosynthétiques et photosynthétiques. L'importance conceptuelle de cette hétérogénéité sera discutée par la suite. Une autre particularité des archaebactéries est l'absence d'une vraie paroi entourant la membrane cellulaire. Une propriété unique des protistes procaryotes est la présence d'une paroi en plusieurs couches pouvant représenter jusqu'à la moitié du poids sec de la cellule. La résistance bactérienne aux variations de pression osmotique du milieu est liée à la paroi, comme le démontre la lyse survenant à la suite de la digestion enzymatique de celle-ci ou à l'inhibition de sa synthèse. La paroi archaebactérienne est fortement réduite par rapport à celle des eubactéries, et dans certains thermophyles, elle est constituée d'un ensemble de monomères protéiques rappelant l'agencement des capsides virales (figure 3 ) [I6]. Une découverte importante pour l'étude de l'évolution a été la mise en évidence d'une hétérogénéité génétique chez les archaebactéries. Rien que dans les ADN des méthanogènes, on a décrit des valeurs du pourcentage molaire en guanine +cytosine (\% GC) allant de 25 à 55 (d'habitude on retrouve des écarts de moins de $5 \%$ à l'intérieur d'une espèce, et de moins de ro \% à l'intérieur d'un genre). Ceci prouve que le royaume archaebactérien renferme un éventail d'espèces presque aussi large que celui des eubactéries, et justifie tout à fait la thèse développée par Woese.

\section{Propriétés biochimiques}

\section{REEFERENCES}

16. Kandler $\mathrm{O}$, König $\mathrm{H}$. Chemical composition of the peptidoglycan-free cell walls of methanogenic bacteria. Arch Microbiol 1978; 1 18: 141-52.

17. Makula RA, Singer ME. Ether-containing lipids of methanogenic bacteria. Biochem Biophys Res Commun 1978; 82: 716-22.

18. Kessel M, Klink F. Archaebacterial elongation factor is ADP-ribosylated by diphtheria toxin. Nature 1980; 287: 250-I.

19. Kessel M, Klink F. Elongation factors of the extremely halnıhilic archaebacterium Halobacterium cutirubrum. Eur 7 Biochem 1981; 1 14:481-6.
Voici certains traits métaboliques et structuraux spécifiques des archaebactéries :

(a) Absence de peptidoglycane*. Le peptidoglycane ou muréine représente la couche majeure de la paroi bactérienne : il s'agit d'un glycopeptide synthétisé par une voie métabolique unique dans la nature.

Chez les archaebactéries, on n'a pas découvert de peptidoglycane ni ses deux constituants de base, l'acide muramique et l'acide diamilique[I6].

* voir glossaire page 438 . (b) Présence de lipides polyisoprénoïdes type éther* La majorité des lipides eubactériens et eucaryotes sont saponifiables (liaison ester d'acides gras linéaires avec le glycérol) : ceux-ci sont remplacés chez les archaebactéries par des phytanols ramifiés et méthylés*, qui sont liés par des liaisons éther au glycérol [17].

(c) Absence de thymine dans l'ARNt. Chez les eubactéries et les eucaryotes, les ARNt contiennent des bases modifiées dont la thymine (en remplacement de l'uracil) : dans les ARNt des archacbactéries, on retrouve la pseudouridine ${ }^{*}$ à la place de la thymine.

(d) Structure unique des ARN ribosomaux (ARNr). L'analyse comparative des $A R N r$ $5 \mathrm{~S}, \mathrm{I} 6 \mathrm{~S}$ et ${ }_{23} \mathrm{~S}$ (certaines de ces molécules ont été séquencées intégralement et leur structure secondaire établie) a révélé l'unicité des $\mathrm{ARNr}$ archaebactériens, qui diffêrent aussi bien des ARNr eubactériens que des molécules homologues retrouvées chez les eucaryotes.

(e) Un ARNt initiateur à méthionine. L'aminoacyl-ARNt, se liant à la sous-unité $30 \mathrm{~S}$ lors de l'initiation de la synthèse protéique, est porteur de formyl-méthionine chez les eubactéries. L'ARNt initiateur porte la méthionine aussi bien chez les archaebactéries que chez les eucaryotes.

(f) Présence d'un facteur d'élongation sensible à la toxine diphtérique. La toxine diphtérique catalyse l'inactivation par ribosylation des facteurs d'élongation eEF-2 des eucaryotes et EFG des archaebactéries, alors que la toxine n'a aucune action sur le facteur d'élongation correspondant EFG des cubactéries [18, 19].

(b) Présence de gènes interrompus. Chez les eucaryotes, les séquences "exprimées" ou exons sont interrompues par des séquences d'intercalation, dites " silencieuses" (les introns).

On retrouve ce phénomène des gènes interrompus également chez les archaebactéries, où des séquences de 15 à 25 paires de bases (séquences comparables aux " introns " des eucaryotes) ont été mises en évidence dans certains gènes. 


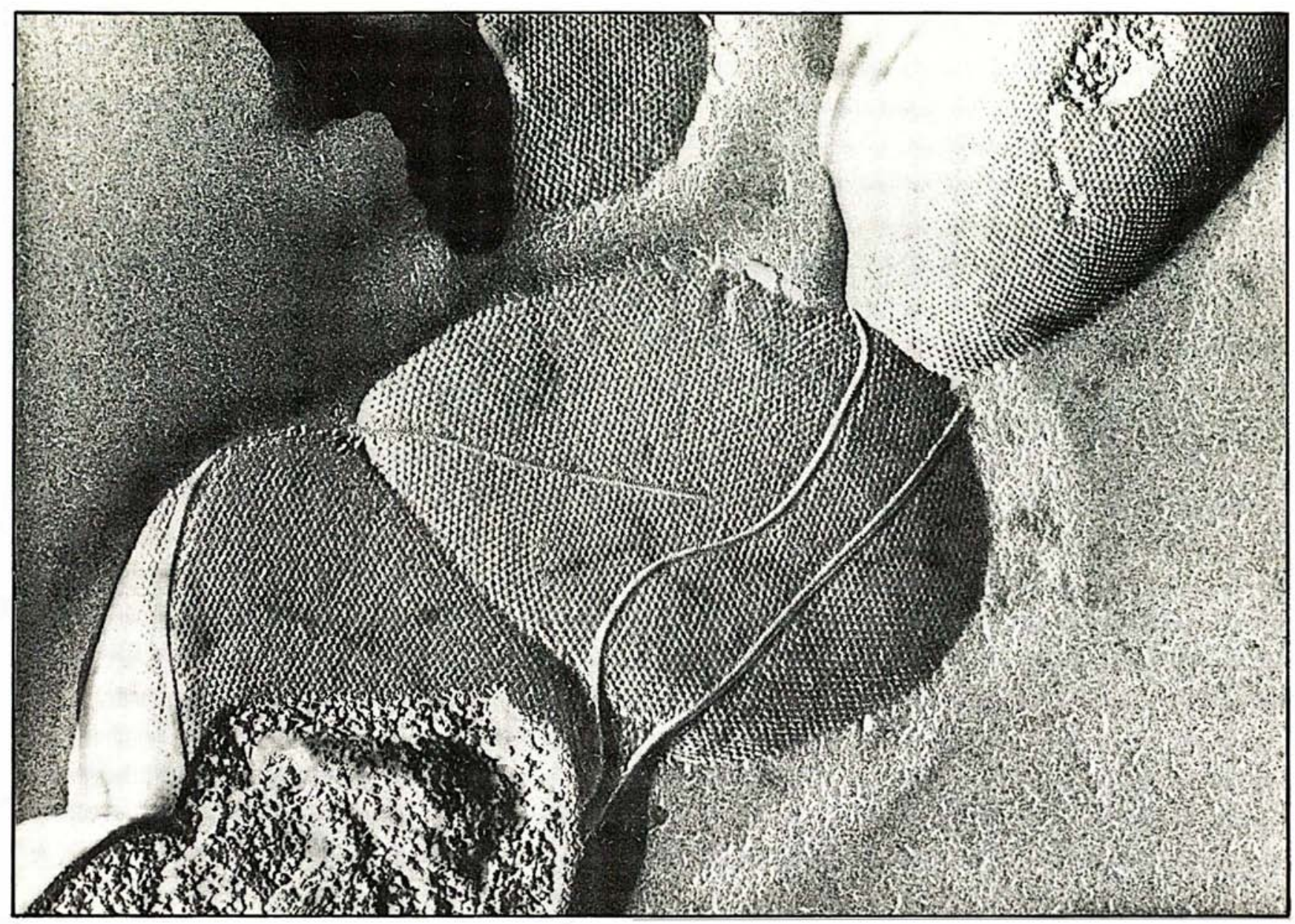

Figure 3. A et B. Micrographies électroniques de Methanogenium marisnigri. Ces remarquables photos prises au microscope électronique à balayage par le Dr Frank Mayer (Institut für Mikrobiologie. Universität Göttingen. Allemagne) montrent la paroi unique d'une archaebactérie méthanogène venant du fond de la Mer Noire. On peut remarquer l'agencement des sous-unités protéiques ressemblant à celui des capsomères viraux. Les agrandissements finaux sont respectivement de 71000 et de 150000 fois.

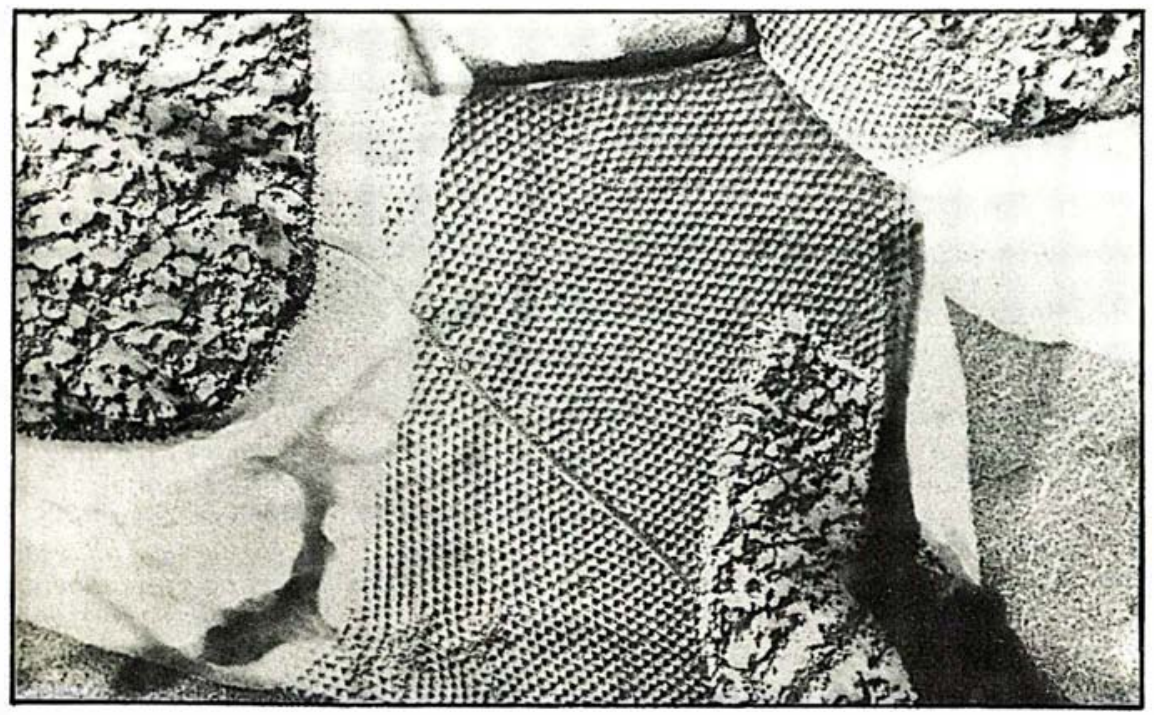

$m / s n^{\circ} 8$, vol. 2, octobre 86 
(h) Promoteurs spéciaux. La transcription des gènes de structure débute par l'attachement de l'ARN polymérase à des promoteurs, qui sont différents pour les eucaryotes, les procaryotes et les archaebactéries*. A ces dernières, en particu- lier, manqueraient les séquences "Shine-Dalgarno "* qui sont spécifiques des eubactéries.

(i) Propriétés singulières des protéines ribosomales (protéines-r). Le nombre des protéines-r est différent chez différents groupes

\section{REFEERENCES}

20. Schmid G, Böck A. The ribosomal protein composition of five methanogenic bacteria. $Z b$ Bakt Hyg I 1982; C 3 : 347-53.

21. Schmid G, Pecher T, Bōck A. Properties of the translational apparatus of archaebacteria. $Z b$ Bakt Hyg I 1982; C 3: 209-17.

22. Hilpert R, Winter J, Hammes W, Kandler O. The sensitivity of archaebacteria to antibiotics. Zbl Bakt Hyg I 198I; C 2: 11-20.

23. Pecher T, Bōck A. In vivo susceptibility of halophilic and methanogenic organisms to protein synthesis inhibitors. FEMS Microbiol Lett $198 \mathrm{I}$; I0: 295-7

24. Elhardt D, Böck A An in vivo polypeptide synthesizing system from methanogenic bacteria: sensitivity to antibiotics. Mol Gen Genet 1982; I88: 1 28-34.

25. Cocito $C$. Antibiotics of the virginiamycin family, inhibitors which contain synergistic components. Microbiol Rev 1 979; 43: 145-98.

26. Cocito C. Properties of virginiamycin-like antibiotics (synergimycins), inhibitors containing synergistic components. In: Hahn FE, Corcoran JW, eds. Antibiotics. Berlin: Springer Verlag, I983: 296-332.

27. Di Giambattista $\mathbf{M}$, Hummel $\mathrm{H}$, Böck $A$ Cocito C. Action of synergimycins and macrolides on in vivo and in vitro protein synthesis in archaebacteria. Mol Gen Genet 1985; 199: 323-9.

28. Woese CR, Fox GE. Phylogenetic structure of the prokaryotic domain: the primary kingdoms. Proc Natl Acad Sci USA 1977; 74: 5088-9o.

29. Balch WE, Magrum LJ, Fox GE, Wolfe RS, Woese CR. An ancient divergence among the bacteria. J Mol Evol 1977; 9: 305-11.

30. Woese CR, Magrum LJ, Fox GE Archaebacteria. J Mol Evol 1978; I1: 245-52.

31. Balch WE, Fox GE, Magrum LJ, Woese CR Wolfe RS. Reevaluation of a unique biological group. Microbiol Rev 1979; 43: 260-96.

32. Lake JA, Henderson E, Oakes M, Clark MW. Eocytes: a new ribosome structure indicates a kingdom with a close relationship to eukaryotes. Proc Natl Acad Sci USA 1984; 81: 3786-90.

33. Henderson E, Oakes M, Clark MW, Lake JA Matheson AT, Zillig W. A new ribosome structure. Science 19d4; 225 : 510-2.

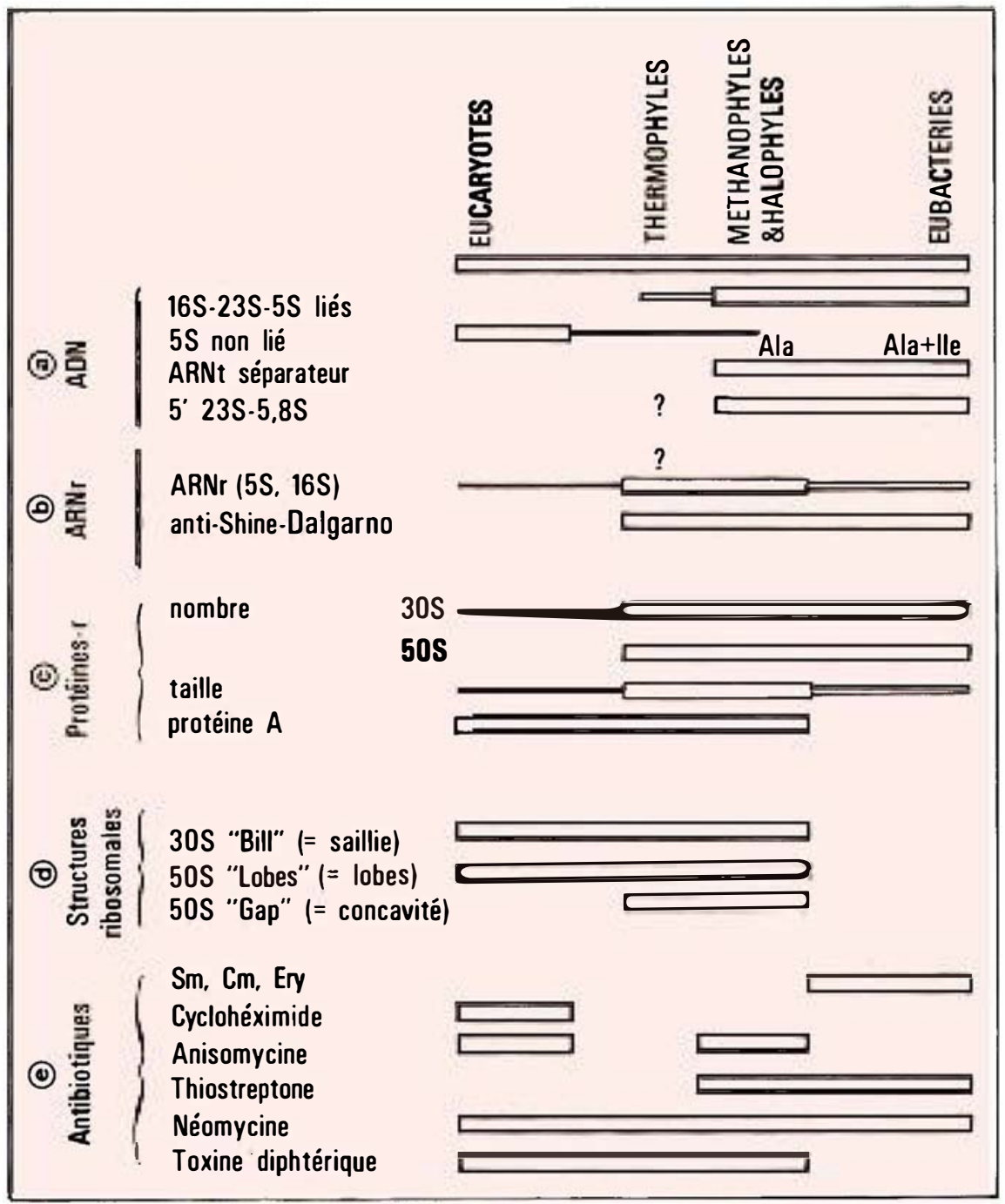

Figure 4. Diagramme des propriétés de l'appareil de traduction des différents organismes. Dans ce diagramme, repris avec modifications de A. Böck, l'existence de propriétés communes entre Eucaryotes (à gauche), Eubactéries (à droite) et Archaebactéries (au centre) est indiquée par un trait dont l'épaisseur est proportionnelle au degré d'homologie. Les paramètres considérés sont: (a) les gènes pour les ARN ribosomaux (ARNr) liés ou non, ainsi que les séquences $5^{\prime}$ de l'ARNr $23 S$ homologues à I'ARN 5,8S des eucaryotes; (b) les structures des ARNr (voir figure 1), ainsi que les séquences hybridant avec le motif de Shine-Dalgarno=anti-Shine-Dalgarno (voir glossaire); (c) le nombre et la taille des protéines-r des sous-unités ribosomales 30 et 50 s; (d) certains traits spécifiques des ribosomes (voir figure 6): (e) la sensibilité à différents inhibiteurs (antibiotiques et toxines) [/e premier groupe comprenant les aminoglycosides (Sm), le chloramphénicol $(\mathrm{Cm}$ ) et les macrolides (Ery) qui sont spécifiques des ribosomes $70 \mathrm{~S}$ et le deuxième groupe la cyclohéximide (Cyc) spécifique des ribosomes $80 S$ ]. Ala =alanine; $/ / e=i s o l e u c i n e$. 
d'archaebactéries par rapport aux eubactérics ( 52 pour l'E. coli, 54 pour Methanococcus et 64 pour Sulfolobus). En outre, les protéines-r des archaebactéries sont moins basiques que celles des eubactéries, et celles des halophiles extrêmes sont nettement acides $[20,2 \mathrm{I}]$. Les $5^{2}$ protéines-r d'E. coli, aujourd'hui entièrement séquencées, pourront servir de référence pour les protéines-r archaebactériennes dont l'analyse est en cours dans plusicurs laboratoires.

(j) "Schéma " unique de sensibilité aux inhibiteurs de la synthèse d'acides nucléiques. Nous citerons, à titre d'exemple, l'inhibi- tion de l'ARN polymérase. L' $\alpha$ amanitine inhibe l'ARN polymérase II des eucaryotes et la rifampicine l'ARN polymérase des cubactéries. Par contre, les archaebactéries sont résistantes aux deux inhibitcurs [22].

(k) Susceptibilité très particulière aux inhibiteurs de la synthèse protéique. L'analyse comparative de la sensibilité déployée par les trois types d'organismes a permis de partager les inhibiteurs de la synthèse protéique en cinq groupes : antibiotiques actifs sculement sur les eubactéries (érythromycine), ou les archacbactéries, ou les cucaryotes (cycloheximide), soit sur

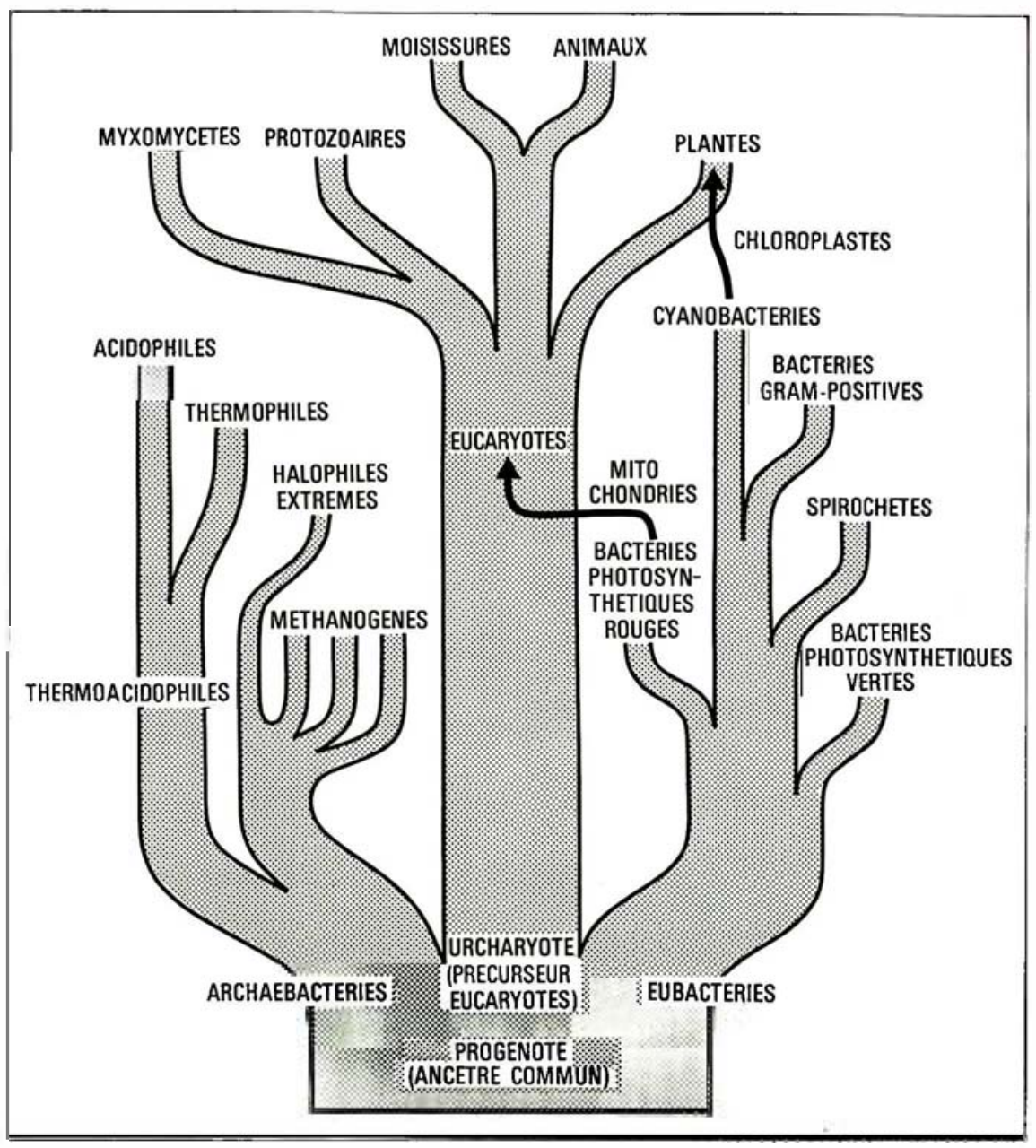

Figure 5. Arbre généalogique des êtres vivants d'après $C$. $R$. Woese. Cet auteur a proposé l'origine (à partir d'un hypothétique ancêtre commun dit progénote) de trois royaumes : les archaebactéries, les eubactéries et les eucaryotes. Le dernier groupe a évolué à partir d'un postulé ancêtre commun. deux groupes (anisomycine pour les cucaryotes et les archacbactéries), soit sur les trois (puromycine) [23, 24]. Chez les eubactéries, on observe un antagonisme entre virginiamycine $\mathrm{A}$ et macrolides, et un synergisme entre virginiamycines A et $B[25,26]$. Les archacbactéries sont insensibles aux trois groupes d'antibiotiques isolés: toutefois, chez les méthanogènes, les virginiamycines $A$ et $B$ ensemble excrcent une action inhibitrice remarquable [27].

L'ensemble de ces données montre clairement que les archaebactéries constituent un groupe d'organismes biochimiquement distinct aussi bien des eubactéries que des eucaryotes ( figure 4).

\section{Arbres généalogiques récents}

Sur la base des données exposées ci-dessus, Woese a proposé un nouveau schéma évolutif, qui prévoit l'origine indépendante des cubactéries-cyanobactéries d'une part, des archaebactéries et des eucaryotes d'autre part (figure , $C$ et figure 5) [28-3r]. Et, en fait, les archaebactéries, tout en partageant avec les eubactéries une organisation cellulaire de type procaryote, diffèrent de celles-ci sur de nombreux points qui les rapprochent, par contre, des cucaryotes. Le schéma de Woese, qui a été confirmé et complété par de nombreuses études au cours de la dernière décennie, a été remis en cause par des travaux récents de Lake et collaboratcurs, consacrés à l'analyse de la morphologie ribosomale par des techniques de microscopie électronique [9]. Selon ces études, les sous-unités ribosomales retrouvées dans la nature peuvent être classécs en quatre types morphologiques, grâce à la présence d'appendices particuliers correspondant à des protéines-r spéciales. Ainsi, la tête de la petite sous-unité présente une protubérance ("bill " $=$ bec) aussi bien dans le cas des archaebactéries que des eucaryotes, mais seulement les organismes sulfo-dépendants partagent avec les eucaryotes la présence de deux protubérances du corps de la $30 \mathrm{~S}$ ("lobes") (figure $6 A)$. De plus, la 

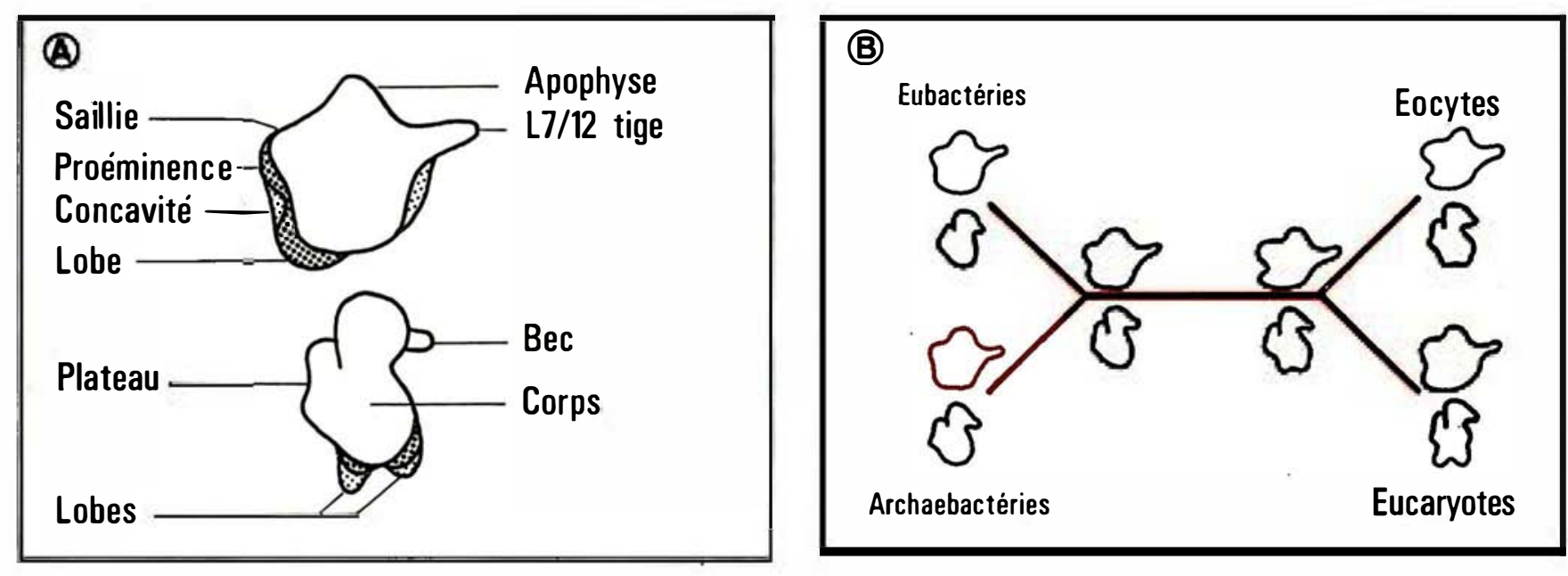

Figure 6. L'évolution des sous-unités ribosomales. A. Structure de la grande sous-unité (partie supérieure) et de la petite sous-unité (partie inférieure). Les structures spécifiques des eucaryotes sont en pointillés fins et celles des éocytes sont en pointillés épais. B. Évolution de la structure ribosomale selon l'arbre esquissé en figure $1 D$. D'après [32] et [33].

grande sous-unité ribosomique possède deux protubérances latérales (" bulge" $=$ proéminence et "lobe $"=$ lobe) aussi bien chez les sulfo-bactéries que chez les eucaryotes (avec la différence que chez les premiers seulement ces protubérances sont séparées par une coupure) (figure $6 B[32,33]$. Les ribosomes des archaebactéries sulfo-dépendantes paraissent donc être des entités uniques, différentes des ribosomes des autres ĉtres vivants. Par ailleurs, ces mêmes sulfo-bactéries ont d'autres propriétés singulières, telles que la présence dans le génome d' introns" semblables à ceux mis en évidence chez les eucaryotes, et l'existence d'ARN polymérases ayant des sous-unités semblables à celles des eucaryotes. En outre, les séquences des ARNr $5 \mathrm{~S}$ et des ARNt initiateurs de Sulfolobus se sont révélées plus proches des molécules correspondantes des eucaryotes que de celles des autres procaryotes. Enfin, les ARN messagers de Sulfolobus possèdent des séquences polyadénylées qui sont caractéristiques des eucaryotes, et qui n'ont pas été retrouvées chez les eubactéries.

Sur la base de toutes ces données,
Lake et collaborateurs ont proposé un nouveau modèle d'arbre évolutif qui voit l'origine, à partir d'un ancêtre commun, de quatre royaumes distincts représentés par les eucaryotes, les cubactéries (incluant les cyanobactéries), les archaebactéries et les éocytes (appelés jusqu'à présent archaebactéries sulfo-dépendantes) (figure $6 \mathrm{~A}$ ) $[32,33]$. Les deux modèles de Woese (1977) et de Lake (1984) sont représentés ensemble, pour comparaison, dans la figure I $D$. Le schéma de Lake indique une séparation tardive entre les éocytes et les eucaryotes d'une part, et entre les archaebactéries et les cubactéries d'autre part (figure I $D$ et $6 \mathrm{~A}$ ).

L'importance des études décrites dans cet article découle du fait qu'elles portent sur une partie considérable de l'âge de la terre. Les micro-organismes, les seuls habitants de notre planète pendant $2,5 \times 10^{9}$ années (les organismes supérieurs ayant existé pendant un laps de temps cinq fois moindre) peuvent être considérés comme une sorte de fossiles vivants, qui portent inscrits dans leurs molécules la longue histoire de l'origine et de l'évolution de la vie

\section{Summary}

Archaebacteria are procaryotes with properties different from those of both eubacteria and cyanobacteria, but with a similar genomic heterogeneity. The three archacbacterial groups (methanogens, halophyles and thermoacidophyles) are found within unusual niches mimicking the Earth $3 \times 10^{9}$ years ago. Ribosomes, high polymers and some low molecular weight compounds isolated from archacbacteria differ from the corresponding molecules of either eucaryotes or cubacteria. The evolutionary tree built on this ground, proposes the origin as independent phyla of eubacteria/cyanobacteria, archacbacteria and urcaryotes: the latter giving rise to cucaryotes through a stable symbiosis with cubacteria (mitochondria) and cyanobacteria (chloroplasts). This scheme has been modified following the discovery of the cocytes (sulfodependent archacbacteria) as an independent group, according to the morphology of ribosomal subunits.

\section{TIRES A PART}

C. Cocito : Unitẻ de microbiologic générale ct de génétique moléculairc, faculté de médecinc, ICP, UCL 7449, avenue Hippocrate 75, B-I 200 Bruxelles. 